\title{
Iterative Interference Cancellation and Channel Estimation for Coded OFDM-CDMA
}

\author{
Volker Kühn \\ Universität Bremen (Germany) \\ Department of Communications Engineering \\ Otto-Hahn-Allee NW 1, D-28359 Bremen \\ kuehn@ant.uni-bremen.de
}

\begin{abstract}
In recent years, spectral efficiency has become a key parameter in mobile radio communications. For Code Division Multiple Access systems, high spectral efficiencies can only be achieved by the application of multi-user detection schemes combatting the inherent multi-user interference. This paper analyzes the system performance of a quasi-synchronous OFDM-CDMA uplink for nonlinear successive interference cancellation (SIC). Realizing that channel estimation is crucial for the performance of interference cancellation schemes, their iterative structure is exploited for improving channel estimation as well as SIC significantly.

First, it is shown that convolutional codes with low memory perform much better for extremely high system loads than codes with large memory. Furthermore, correlative, maximum likelihood (ML) channel estimation as well as iteratively improved ML estimation are compared. Simulation results demonstrate that the loss compared to the single-user bound with perfect channel knowledge amounts only $0.7 \mathrm{~dB}$ even for loads of $\beta=2$, QPSK modulation and real channel estimation.
\end{abstract}

\section{INTRODUCTION}

Code Division Multiple Access (CDMA) has become a widely accepted multiple access technique in mobile radio communications. One attractive realization is OFDM-CDMA (Orthogonal Frequency Division Multiplex) where each chip is only affected by flat fading [1]-[3]. For the uplink transmission, the orthogonality of spreading codes cannot be maintained and random spreading sequences are used. Hence, multi-user interference is the limiting factor concerning system capacity. However, spectral efficiency can be increased by applying multi-user detection (MUD) techniques. In the last years, plenty of work has been spent on this topic, especially on iterative MUD techniques [4]-[12]. Furthermore, capacity bounds have been analytically derived indicating the maximum system load that should be theoretically reachable [13]-[15].

This paper considers the uplink of a coded OFDM-CDMA system with BPSK and QPSK modulation. A coarse synchronization of all active users which is under discussion for future mobile radio systems is presupposed leading to a quasisynchronous system that allows efficient realizations of multiuser detection schemes. In order to combat multi-user interference, nonlinear successive interference cancellation (SIC) is applied. In this context, channel estimation is a critical task especially for extremely high system loads. Therefore, the combination of channel estimation and successive interference cancellation is considered.
First, it is demonstrated that strong convolutional codes with large memory perform worse than short memory codes when SIC is applied at extremely high loads. Second, different channel estimation techniques are examined, namely simple correlative and maximum likelihood (ML) estimation as well as an iterative approach improving the channel estimates in each iteration of the SIC. While the first two estimation techniques lead not to a convergence of the interference cancellation scheme due to more or less bad channel estimates, the latter one has the potential to reach the single-user bound even for a system load of $\beta=2$.

The paper is structured as follows: Section 2 describes the coded OFDM-CDMA system. Next, section 3 presents the ingredients of the successive interference cancellation scheme and discusses its performance for perfect channel estimation. Section 4 then derives different channel estimation techniques and demonstrates their performances by some simulation results. Section 5 summarizes the main results.

\section{SySTEM DESCRIPTION}

\section{A. OFDM-CDMA System}

The structure of the considered OFDM-CDMA system is depicted in Fig. 1. We assume a frame oriented transmission where each user $u, 1 \leq u \leq U$, transmits a sequence of information bits $\mathbf{d}_{u}$ of length $L_{d}$. They are first encoded by identical convolutional codes of rate $R_{c}=1 / n$ and constraint length $L_{c}$. The resulting vectors $\mathbf{b}_{u}$ are interleaved by userspecific interleavers $\Pi_{u}$ of length $L_{b}=n L_{d}$ and $M$-PSK modulated, i.e. groups $\mathbf{b}_{u}(\ell)=\left[b_{u, 1}(\ell) \cdots b_{u, m}(\ell)\right], 0 \leq \ell<$ $L_{b} / m$, of $m$ bits are mapped onto one of $M=2^{m}$ PSK symbols. Next, direct-sequence spreading by a factor $N_{s}$ is carried out with pseudo random long codes $\mathbf{c}_{u} \in\{ \pm 1 / \sqrt{2} \pm$ $j / \sqrt{2}\}^{L_{x}}, L_{x}=L_{d} n N_{s} / m$. The system load as one of the key parameters is defined by $\beta=U / N_{s}$.

Finally, the OFDM transmitter transforms the obtained vector $\mathbf{x}_{u}$ into the time domain. In this work, the number of carriers $N_{c}$ equals $n N_{s}$, i.e. $m$ information bits or equivalently $n$ PSK symbols are mapped onto one OFDM symbol. After interleaving in frequency domain over $N_{c}$ chips and an inverse Fourier transformation, a cyclic prefix of duration $T_{g}$ called guard interval is inserted in front of each OFDM symbol [3]. This prefix guarantees a cyclic convolution between the core OFDM symbol and the channel impulse response resulting in 


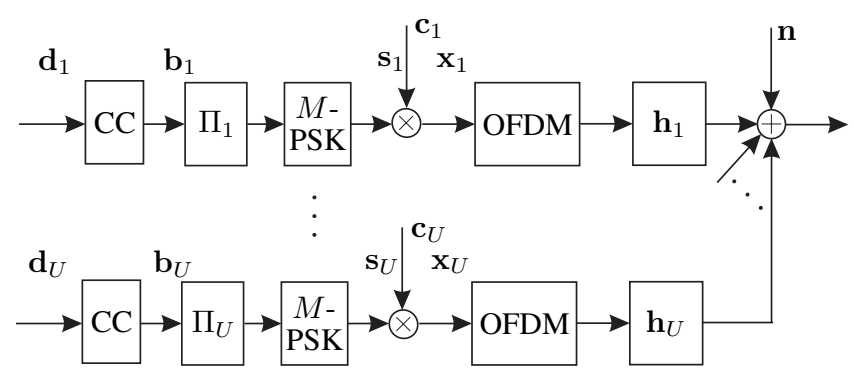

Fig. 1. Structure of coded OFDM-CDMA system

a scalar multiplication in the frequency domain. Hence, each chip is only affected by flat fading.

\section{B. Channel Model}

The signals of all users are now transmitted over $J$ individual $L$-path block fading channels, i.e. the channels remain constant during one fading block consisting of $L_{f}$ OFDM symbols. Channel impulse responses of successive fading blocks (time index $k$ ) are statistically independent. Real and imaginary parts of the corresponding channel coefficients $h_{u, l}(k), 0 \leq l<L$, are gaussian distributed and statistically independent. Although each user is assigned to an individual channel, the number of transmission paths $L$ is assumed to be the same for all users. The corresponding transfer functions are defined by

$$
H_{u, \mu}(k)=\sum_{l=0}^{L-1} h_{u, l}(k) \cdot e^{-j 2 \pi \mu l / N_{c}} .
$$

At the receiver, the cyclic prefix is removed first. Assuming rough synchronization, i.e. the maximum delay between different users is limited to the difference between guard time and maximum delay of the user-specific channels, one FFT window suffices for transforming all user signals back into the frequency domain. The obtained vector $\mathbf{r}$ at the output of the OFDM receiver is now fed to the successive interference canceller described in section III.

\section{Simulation Parameters}

In order to evaluate the performance of the OFDM-CDMA system, simulations were carried out with the following parameters. Two different convolutional codes of rate $R_{c}=1 / 2$ are employed, an $L_{c}=3$ code with generator polynomials $\left[1+D+D^{2}, 1+D^{2}\right](\mathrm{CC} 3)$ and an $L_{c}=7$ code with $\left[1+D^{2}+D^{3}+D^{5}+D^{6}, 1+D+D^{2}+D^{3}+D^{6}\right]$ (CC7). Furthermore, BPSK $(M=2)$ and QPSK $(M=4)$ have been used for PSK modulation. CDMA spreading was performed by a factor $N_{s}=32$ so that the number of sub-carriers of one OFDM symbol amounts $N_{c}=n N_{s}=64$.

As mobile radio channel, a 4-paths Rayleigh fading channel with equal average power on each tap was used. Assuming a signal bandwidth of $B=5 \mathrm{MHz}$, the duration of an OFDM symbol is $T_{s}=12.8 \mu \mathrm{s}$. Different fading rates were considered by varying the lengths of the fading blocks. Fast fading channels with a maximum Doppler frequency of $f_{d \max }=200 \mathrm{~Hz}$
TABLE I

LIST OF INTERLEAVER LENGTH FOR DIFFERENT FADING RATES

\begin{tabular}{l|c|c|c|c}
$f_{d \max }$ & $L_{f}$ & $L_{p}$ & BPSK & QPSK \\
\hline $40 \mathrm{~Hz}$ & 20 & 4 & 224 & 448 \\
\hline $100 \mathrm{~Hz}$ & 40 & 8 & 448 & 896 \\
\hline $200 \mathrm{~Hz}$ & 100 & 8 & - & 2576 \\
& & 20 & - & 2240
\end{tabular}

(corresponding to a coherence time of $T_{c}=2.5 \mathrm{~ms}$ ) result in block length of $L_{f}=20$ OFDM symbols. Slow fading channels with $f_{d \max }=40 \mathrm{~Hz}$ and $T_{c}=12.5 \mathrm{~ms}$ lead to $L_{f}=100$. An intermediate channel with $L_{f}=40\left(f_{d \max }=100 \mathrm{~Hz}\right)$ was also chosen.

In order to ensure equal diversity degrees for all configurations, the number of fading blocks within a data frame is kept constant at $N_{f}=7$. Consequently, the interleavers' lengths depend on the fading block lengths and the type of modulation. They are listed in Table I.

Concerning channel estimation, the following frame structure was chosen. The data frame consists of $L_{s}=L_{d} / \mathrm{m}$ OFDM symbols and is divided into $N_{f}$ sub-frames according to the number of fading blocks. Each of these sub-frames comprises a pilot sequence $\mathbf{P}_{u}(k)$ of $L_{p}$ OFDM symbols and the corresponding part of the data sequence of length $L_{f}-L_{p}=L_{s} / N_{f}$. The relative redundancy introduced by the pilots is $L_{p} / L_{f}=20 \%$ (except for $L_{p}=8$ and $L_{f}=100$ ) and therefore comparable to GSM systems [16].

\section{Nonlinear Successive Interference CANCELlation}

\section{A. Receiver Structure}

First, vector $\mathbf{r}$ at the output of the OFDM receiver is processed by a bank of single-user matched filters performing the despreading (maximum ratio combining) [3]. Based on the resulting signals $\hat{\mathbf{s}}_{u}, \log$-likelihood values are calculated for each of the $m$ bits of a PSK symbol $\hat{s}_{u}(\ell), 0 \leq \ell<L_{b} / m$

$$
\hat{b}_{u, \nu}(\ell)=\log \frac{\sum_{s \in \mathcal{S}_{\nu}^{+}} P\left(\hat{s}_{u}(\ell) \mid s\right)}{\sum_{s \in \mathcal{S}_{\nu}^{-}} P\left(\hat{s}_{u}(\ell) \mid s\right)}, 1 \leq \nu \leq m .
$$

In (2), $\mathcal{S}_{\nu}^{+}$and $\mathcal{S}_{\nu}^{-}$denote the sets of PSK symbols that correspond to $b_{u, \nu}(\ell)=+1$ and $b_{u, \nu}(\ell)=-1$, respectively ${ }^{1}$. After subsequent de-interleaving, symbol-by-symbol soft-in/soft-out decoding is applied by a Max-Log-MAP decoder [17] that approximates log-likelihood values of the coded bits

$$
\lambda_{u, \nu}(\ell)=\log \frac{P\left(b_{u, \nu}(\ell)=+1 \mid \hat{\mathbf{b}}_{u}\right)}{P\left(b_{u, \nu}(\ell)=-1 \mid \hat{\mathbf{b}}_{u}\right)}
$$

\footnotetext{
${ }^{1}$ For QPSK modulation, (2) reduces to $\hat{b}_{u, 1}(\ell)=\gamma \operatorname{Re}\left\{\hat{s}_{u}(\ell)\right\}$ and $\hat{b}_{u, 2}(\ell)=\gamma \operatorname{Im}\left\{\hat{s}_{u}(\ell)\right\}$ where $\gamma$ is the reliability of the channel. For BPSK, only the real part has to be considered.
} 
and delivers decisions of the information bits $\hat{\mathbf{d}}_{u}$. In order to reconstruct the transmitted signals for interference cancellation, soft re-modulation is implemented by calculating the expectation

$$
\tilde{s}_{u}(\ell)=\sum_{s \in \mathcal{S}} s \cdot P(s)
$$

where $P(s)$ denotes the a priori probability of a symbol $s$. It can be obtained from the log-likelihood ratios in (3) (omitting the time index $\ell$ ) by

$$
P(s)=\prod_{\nu=1}^{m} \frac{\exp \left(\lambda_{u, \nu} / 2\right)}{1+\exp \left(\lambda_{u, \nu}\right)} \cdot \exp \left(a_{\nu}(s) \lambda_{u, \nu} / 2\right)
$$

with $a_{\nu}(s)= \pm 1$ representing the $\nu$-th bit within the binary representation of a PSK symbol $s .^{2}$ With (4), the transmitted sequences can now be reconstructed. After re-spreading (yielding $\tilde{\mathbf{x}}_{u}$ ) and weighting with the corresponding channel transfer function $\mathbf{H}_{u}$, the sequences $\tilde{\mathbf{r}}_{u}$ represent the estimated received sequences of different users $u$. They are used to cancel interference successively from the received signal $\mathbf{r}$ and, therefore, improve the outputs of the matched filter bank ${ }^{3}$.

Starting with user $u=1$, the interference reduced signal for user $u$ in iteration step $\mu$ can be expressed as

$$
\tilde{\mathbf{r}}_{u}^{(\mu)}=\mathbf{r}-\sum_{u^{\prime}=1}^{u-1} \tilde{\mathbf{r}}_{u^{\prime}}^{(\mu)}-\sum_{u^{\prime}=u+1}^{U} \tilde{\mathbf{r}}_{u^{\prime}}^{(\mu-1)} .
$$

For linear cancellation schemes, (6) is also well-known as Gauss-Seidel iteration.

\section{B. Results for Perfect Channel Estimation}

Figure 2 shows the results for an OFDM-CDMA system with perfect channel knowledge and $U=64$ active users. This corresponds to a system load of $\beta=2$. For BPSK, it can be observed that the stronger convolutional code with constraint length $L_{c}=7$ reaches the single-user bound (SUB) after 4 iterations and outperforms the weaker code by $2 \mathrm{~dB}$ at a BER of $10^{-5}$. The $L_{c}=3$ code approaches the SUB to less than $0.2 \mathrm{~dB}$ (even with further iterations).

However, for QPSK modulation, the system load is doubled and the $L_{c}=7$ code is far away from reaching the single user performance even after 10 iterations. Even in this case, the $L_{c}=3$ code is still able to nearly remove the interference within 10 iterations so that the single-user bound is reached within $0.3 \mathrm{~dB}$. It has to be mentioned that the computational decoding costs amount only $1 / 16$ compared to the $L_{c}=7$ code.

These results can be explained by having a look at the EXIT charts [18] of both codes. At very low signal-to-noise ratios the weaker code delivers more mutual information about the coded bits than the stronger code resulting in a better convergence of the SIC loop.

\footnotetext{
${ }^{2}$ For QPSK, (4) reduces to $\tilde{s}_{u}(\ell)=\tanh \left(\lambda_{u, 1} / 2\right)+j \tanh \left(\lambda_{u, 2} / 2\right)$.

${ }^{3}$ This is the same procedure as described in [10] except that not only the extrinsic part but the entire decoder output is used for interference cancellation.
}

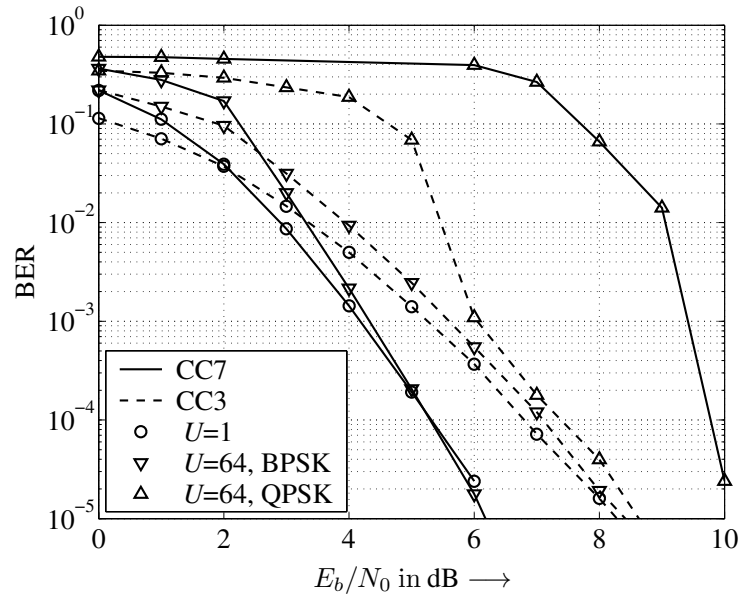

Fig. 2. BER performance of different convolutional codes for $U=64$ users $(\beta=2)$ and perfect channel knowledge (BPSK: 4 iterations, QPSK: 10 iterations)

\section{Channel Estimation}

\section{A. Mathematical Description}

The results of the previous section have been obtained with the assumption of perfect channel knowledge. However, accurate channel estimation is a critical task especially in the case of severe multi-user interference. Bad estimates with phase errors of $\pi$ would result in an interference amplification instead of a reduction. Therefore, we propose a scheme where initial channel estimates are updated in each iteration exploiting the decoder outputs. A similar strategy was recently proposed in [5], [10] as EM-based approach (EM: Expectation Maximization).

The received vector $\mathbf{r}$ at the output of the OFDM receiver is divided into sub-frames according to the frame structure described in section II. In the sequel, we focus on the description for a single fading block and denote its training part with a superscript ${ }^{p}$. The received preambles of all users can be expressed with

$$
\mathbf{r}^{p}=\mathbf{P H}+\mathbf{n}^{p}
$$

where

$$
\mathbf{P}=\left(\begin{array}{ccc}
\operatorname{diag}\left\{\mathbf{P}_{1}(1)\right\} & \cdots & \operatorname{diag}\left\{\mathbf{P}_{U}(1)\right\} \\
\vdots & & \vdots \\
\operatorname{diag}\left\{\mathbf{P}_{1}\left(L_{p}\right)\right\} & \cdots & \operatorname{diag}\left\{\mathbf{P}_{U}\left(L_{p}\right)\right\}
\end{array}\right)
$$

is a $L_{p} U \times N_{c} U$ matrix containing as diagonal sub-matrices $\operatorname{diag}\left\{\mathbf{P}_{u}(i)\right\}$ the $i$-th OFDM pilot symbol of user $u$. The column vector $\mathbf{H}$ comprises the channel transfer functions of all users and $\mathbf{n}^{p}$ represents the background noise during the preamble. Since only $L$ channel coefficients have to be estimated for each user but $N_{c}$ statistically dependent coefficients represent the channel transfer function, it is advantageous to perform the estimation in the time domain. The $N_{c} U \times L U$ block diagonal transformation matrix 


$$
\mathbf{T}_{\mathrm{DFT}}=\left(\begin{array}{ccc}
\text { DFT } & & \\
& \ddots & \\
& & \text { DFT }
\end{array}\right)
$$

consists of $U$ identical $N_{c} \times L$ sub-matrices DFT. With $\mathbf{H}=$ $\mathbf{T}_{\mathrm{DFT}} \cdot \mathbf{h}$, (7) becomes

$$
\mathbf{r}^{p}=\mathbf{P} \mathbf{T}_{\mathrm{DFT}} \mathbf{h}+\mathbf{n}^{p} .
$$

The column vector $\mathbf{h}$ now incorporates the $L U$ channel coefficients to be estimated. For the sake of simplicity, we define the matrix $\tilde{\mathbf{P}}:=\mathbf{P T}_{\mathrm{DFT}}$.

\section{Pilot Based Correlative Channel Estimation}

The simplest method for channel estimation is to correlate $\mathbf{r}^{p}$ with the preambles $\tilde{\mathbf{P}}_{u}$ of each user $u$. We obtain

$$
\hat{\mathbf{h}}_{\text {corr }}=\frac{1}{L_{p}} \tilde{\mathbf{P}}^{H} \mathbf{r}^{p}=\frac{1}{L_{p}} \tilde{\mathbf{P}}^{H} \tilde{\mathbf{P}} \mathbf{h}+\frac{1}{L_{p}} \tilde{\mathbf{P}}^{H} \mathbf{n}^{p} .
$$

The corresponding transfer functions are obtained by $\hat{\mathbf{H}}_{\text {corr }}=\mathbf{T}_{\mathrm{DFT}} \hat{\mathbf{h}}_{\text {corr }}$. Since $\tilde{\mathbf{P}}^{H} \tilde{\mathbf{P}}$ is not diagonal, $\hat{\mathbf{h}}_{\text {corr }}$ suffers not only from the background noise but also from multi-user interference.

\section{Pilot Based ML estimation (ML)}

The maximum likelihood estimation is obtained with the approach $\hat{\mathbf{h}}_{\mathrm{ML}}=\arg \min _{\tilde{\mathbf{h}}}\left\|\mathbf{r}^{p}-\tilde{\mathbf{P}} \tilde{\mathbf{h}}\right\|^{2}$ whose solution is

$$
\hat{\mathbf{h}}_{\mathrm{ML}}=\left(\tilde{\mathbf{P}}{ }^{H} \tilde{\mathbf{P}}\right)^{-1} \tilde{\mathbf{P}}^{H} \mathbf{r}^{p}=\mathbf{h}+\left(\tilde{\mathbf{P}}^{H} \tilde{\mathbf{P}}\right)^{-1} \tilde{\mathbf{P}}^{H} \mathbf{n}^{p}
$$

if $\tilde{\mathbf{P}}^{H} \tilde{\mathbf{P}}$ has full rank. A comparison of (12) with (11) shows that the ML approach incorporates the correlative estimation in a first stage followed by a decorrelation. The calculation of $\tilde{\mathbf{P}}^{H} \tilde{\mathbf{P}}$ can be efficiently realized by

$$
\tilde{\mathbf{P}}^{H} \tilde{\mathbf{P}}=\sum_{i=1}^{L_{p}} \tilde{\mathbf{P}}(i)^{H} \cdot \tilde{\mathbf{P}}(i)
$$

with $\tilde{\mathbf{P}}(i)=\left[\operatorname{diag}\left\{\mathbf{P}_{1}(i)\right\} \mathbf{D F T} \cdots \operatorname{diag}\left\{\mathbf{P}_{U}(i)\right\} \mathbf{D F T}\right]$. Its inverse is approximated by iteratively solving the underlying linear equation system with the Gauss-Seidel algorithm. This solution with $\mu$ iterations demands only an effort of $\mathcal{O}\left(\mu(L U)^{2}\right)$ instead of $\mathcal{O}\left((L U)^{3}\right)$ for a direct matrix inversion. Since $\mu \ll L U$, solving the linear equation iteratively represents an efficient realization of (12) allowing a simple trade-off between computational costs and performance. Although the receiver knows the preambles a priori and can, therefore, pre-calculate and store the inverse in a look-up table, this is not possible for the improved ML approach described below.

\section{Improved ML estimation ( $i M L)$}

Although the estimate in (12) is unbiased it suffers from the amplification of the background noise especially for high system loads and short preambles. Since successive interference cancellation works iteratively, it is obvious to refine the channel estimates during each iteration. Hence, we propose an improved strategy with the ML approach for an initial estimation.

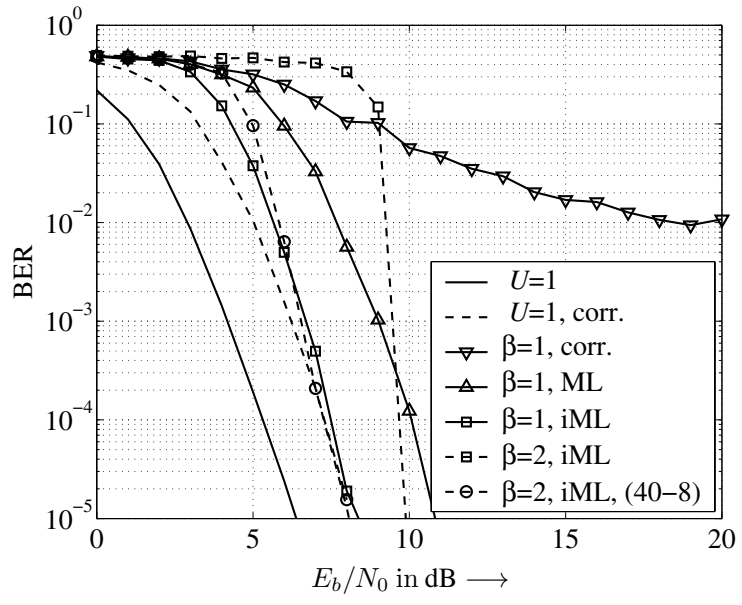

Fig. 3. BER performance for BPSK, CC7, fast fading channels with $L_{f}=20$ (40) and $L_{p}=4$ (8) pilot symbols, 4 SIC iterations

First, the reconstructed sequences $\tilde{\mathbf{x}}_{u}$ obtained at the end of each SIC iteration are used as pseudo-reference symbols for a refined estimation that exploits now the whole sub-frame. Therefore, (12) is not restricted to the preamble and (13) is extended to $1 \leq i \leq L_{f}$. For $L_{p}<i \leq L_{f}, \tilde{\mathbf{P}}_{u}^{(\mu)}(i)$ in the $\mu$-th SIC iteration is obtained by

$$
\tilde{\mathbf{P}}_{u}^{(\mu)}(i)=\operatorname{diag}\left\{\tilde{\mathbf{x}}_{u}^{(\mu)}(i)\right\} \cdot \mathbf{D F T}
$$

where $\tilde{\mathbf{x}}_{u}^{(\mu)}(i)$ denotes the $i$-th reconstructed spread PSK symbol of user $u$ (cf. section III-A).

\section{B. Simulation Results}

Fig. 3 depicts the results for the strong convolutional code (CC7), BPSK modulation, a load of $\beta=1$ and sub-frames of length $L_{f}=20$ with $N_{p}=4$ pilot symbols. For simple correlative channel estimation the interference cannot be removed efficiently and we obtain an error floor at $10^{-2}$. The ML approach leads to better performance and no error floor is visible in the considered range. However, there still remains a gap to the single-user bound of nearly $4.5 \mathrm{~dB}$. A deeper analysis shows that there is no performance improvement after the second SIC iteration because the channel estimates remain inaccurate. Repeating the ML estimation in each SIC iteration using (14) now leads to improvements in each SIC iteration so that the single-user bound for correlative estimation is reached. However, a loss of approximately $2 \mathrm{~dB}$ compared to perfect channel estimation still remains.

Increasing the load to $\beta=2$ and the number of SIC iterations to 8 enlarges the gap to the single-user bound to $3.5 \mathrm{~dB}$. Here, longer sub-frames and therefore slower fading are necessary. With $L_{p}=8$ and $L_{f}=40$ the single-user bound is reached again after 4 iterations. For these high loads and QPSK modulation, the memory 2 code is advantageous. Fig. 4 illustrates the corresponding results for BPSK modulation and different values of $L_{f}$ and $L_{p}$. Even for fast fading channels with short sub-frames and only $L_{p}=4$ pilots the loss compared to the SUB for perfect channel estimation is only 


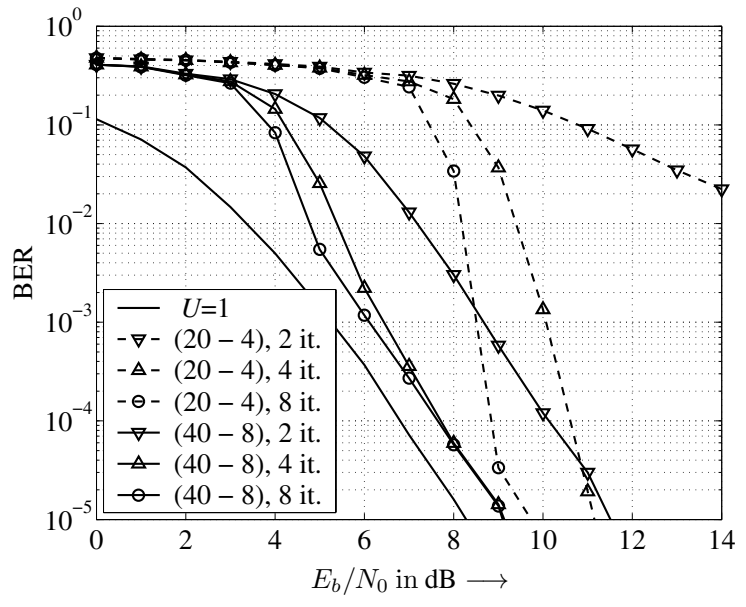

Fig. 4. BER performance for BPSK, CC3, load $\beta=2$ and different $\left(L_{f}-\right.$ $L_{p}$ ) combinations

$1.4 \mathrm{~dB}$. If the channel fades more slowly and, thus, allows longer sub-frames ( $L_{f}=40$ with $L_{p}=8$ ), the gap to the SUB is only $0.7 \mathrm{~dB}$ over a wide range of signal to noise ratios.

Employing QPSK modulation doubles the load because the quadrature component now contains information and, hence, contributes to the multi-user interference. As can be seen in Fig. 5, the performance can be enhanced by increasing $L_{p}$ as well as $L_{f}$. While $L_{p}=8$ pilot symbols seem not to be enough in order to ensure a reliable initial channel estimation, the SUB is reached within $0.7 \mathrm{~dB}$ for $L_{p}=20$ and $L_{f}=100$. Finally, it has to be mentioned that the Gauss-Seidel approximation of the inverse of (13) with only 2 iterations (GS 2) achieves a slightly better result as with 8 iterations and therefore the complexity is not much higher than for simple correlative estimation.

\section{CONClusion}

It was shown that successive interference cancellation can significantly improve the spectral efficiency of OFDM-CDMA systems. For extremely high system loads, convolutional codes with low memory perform better than codes with large memory. The single-user bound could be asymptotically reached within $0.3 \mathrm{~dB}$ for a system load of 2 and QPSK modulation.

However, accurate channel estimation is a necessary precondition for a good convergence of the SIC loop. Systems based only on an initial channel estimation fail to reach the single-user bound. Instead, the iterative structure of the SIC has to be exploited and the channel estimates must be refined in each iteration. Then, the gap to the single-user bound can be reduced to $0.7 \mathrm{~dB}$ for $\beta=2$ and QPSK modulation.

\section{REFERENCES}

[1] S. Kaiser. Multi-Carrier CDMA Mobile Radio Systems - Analysis and Optimization of Detection, Decoding and Channel Estimation. VDI, Januar 1998.

[2] A. Dekorsy and K.D. Kammeyer. A new OFDM-CDMA Uplink Concept with M-ary Orthogonal Modulation. European Transactions on Telecommunications (ETT), 10(4):377-390, July/August 1999.

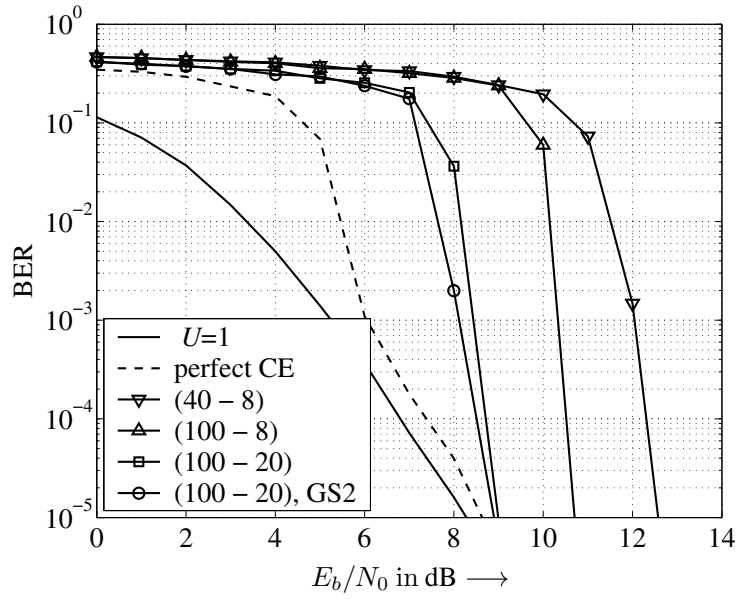

Fig. 5. BER performance for QPSK, CC3, load $\beta=2,10$ SIC iterations and different $\left(L_{f}-L_{p}\right)$ combinations

[3] V. Kühn. Combined MMSE-PIC in Coded OFDM-CDMA Systems. In IEEE Global Conference on Telecommunications (Globecom 2001), San Antonio, USA, November 2001.

[4] S. Moshavi. Multi-User Detection for DS-CDMA Communications. IEEE Communications Magazine, pages 124-136, Oktober 1996.

[5] L.B. Nelson and V. Poor. Iterative Multiuser Receivers for CDMA Channels: An EM-based Approach. IEEE Transactions on Communications, 44(12):1700-1710, December 1996.

[6] S. Verdu. Multiuser Detection. Cambridge University Press, New York, 1998.

[7] M.C. Reed, C.B. Schlegel, P.D. Alexander, and J.A. Asenstorfer. Iterative Multiuser Detection for CDMA with FEC: Near-Single-User Performance. IEEE Transactions on Communications, 46(12):16931699, December 1998.

[8] P.D. Alexander, M.C. Reed, J.A. Asenstorfer, and C.B. Schlegel. Iterative Multiuser Interference Reduction: Turbo CDMA. IEEE Transactions on Communications, 47(7):1008-1014, Juli 1999.

[9] M. Honig and M.K. Tsatsanis. Multiuser CDMA Receivers. IEEE Signal Processing Magazine, pages 49-61, May 2000.

[10] M. Kobayashi, J. Boutros, and G. Caire. Successive Interference Cancellation with SISO Decoding and EM Channel Estimation. IEEE Journal on Selected Areas in Communications, 19(8):1450-1460, August 2001.

[11] A. Lampe, R. Schober, W. Gerstacker, and J. Huber. A Novel Multiuser Detector for Complex Modulation Schemes. IEEE Journal on Selected Areas in Communications, 20(2):339-349, February 2002.

[12] A. Lampe and J. Huber. Iterative Interference Cancellation for DSCDMA Systems with High System Loads Using Reliability-Dependent Feedback. IEEE Transactions on Vehicular Technology, 51(3):445-452, May 2002.

[13] S. Verdu and S. Shamai. Spectral Efficiency of CDMA with Random Spreading. IEEE Transactions on Information Theory, 45(2):622-640, März 1999.

[14] P. Schramm and R.R. Müller. Spectral Efficiency of CDMA Systems with Linear MMSE Interference Suppression. IEEE Transactions on Communications, 47(5):722-731, May 1999.

[15] D. Tse and S.V. Hanly. Linear Multiuser Receivers: Effective Interference, Effective Bandwidth and User Capacity. IEEE Transactions on Information Theory, 45(2):641-657, März 1999.

[16] M. Mouly and M.B. Pautet. The GSM-System for Mobile Communications. Eigenverlag, 1992.

[17] P. Robertson, E. Villebrun, and P. Hoeher. A Comparison of Optimal and Sub-Optimal MAP Decoding Algorithms Operating in the Log-Domain. In International Conference on Communications (ICC'95), pages 10091013, Seattle, USA, June 1995.

[18] S. ten Brink. Code Characteristic Matching for Iterative Decoding of Serially Concatenated Codes. In Ann. des Telecommunication, pages 100-110, 2001 